\title{
Simple devices for restricting the visual fields of birds
}

\author{
JOSH WALLMAN and CHARLOTTE LEDOUX \\ Biology Department \\ City College of the City University of New York, New York, New York 10031 \\ and \\ MARK B. FRIEDMAN \\ Carnegie-Mellon University, Pittsburgh, Pennsylvania 15213
}

\begin{abstract}
The construction and use of several simple occluders designed for long-term use is described. They permit limiting vision either to one eye or to the frontal or lateral visual fields and permit rapid interchange among these conditions.
\end{abstract}

Frequently in behavioral studies it is desirable to limit a bird's vision either to one eye or to one part of the visual field. A variety of hoods (Chiu, Lauber, \& Kinnear, 1975; Hess, 1956; Rossi, 1968; Siegel, 1953), goggles (Catania, 1963), patches (Cherkin, 1970; Lauber, McGinnis, \& Boyd, 1965), and contact lenses (Zeier, 1970) have been used for this purpose. In our experience with young chicks, adult chickens, and ring doves, we have found that the most successful devices for long-term use are those that are lightweight, have no protruding edges, and are securely glued to the skin and feathers. Collodion is an excellent adhesive in this application, since it dries quickly and imbeds both the occluder rim and the surrounding feathers, forming a smooth, well anchored, rigid surface.

We describe here the manufacture and use of two basic types of occluders, each of which with minor modifications either deprives an eye of form vision or restricts vision to one part of the visual field. The first type is lightweight plastic, suitable for long-term use. The second type of occluder is metal. These occluders are heavier but permit rapid interchange of the eye being deprived or the field of view being occluded.

\section{PLASTIC OCCLUDERS}

The general procedure involves heat stamping hemispherical occluders from sheets of translucent vinyl. The stamping device consists of an aluminum rod. An aluminum ball is attached to one end of the rod. The diameter of the ball corresponds to the inner diameter of the occluder. At the other end of the rod,

This work was supported by the City University of New York Faculty Research Award Program and a National Institutes of Health Biomedical Sciences support subgrant. a smooth knob facilitates applying pressure with the palm of the hand. This rod is encased by a sliding brass sleeve that, at the ball end, widens to form a collar that holds the outer edges of the vinyl and prevents pleating of the occluder rim (Figure 1). The mold into which the vinyl is pressed is a cylindrical piece of brass with a bored hole. The radius of the hole is equal to the radius of the aluminum ball plus the thickness of the plastic. (A cruder version of these tools can be made by using a stack of washers taped together for the mold and by soldering another washer or a nut of the same diameter to a piece of brass tubing to form the rim straightener. For the ball one can use part of a balland-socket joint.)

One-inch squares of .02-in.-thick white vinyl are roughened on one side with steel wool to increase opacity and reduce reflection on the inside of the occluder. The cylinder mold is placed in a beaker filled
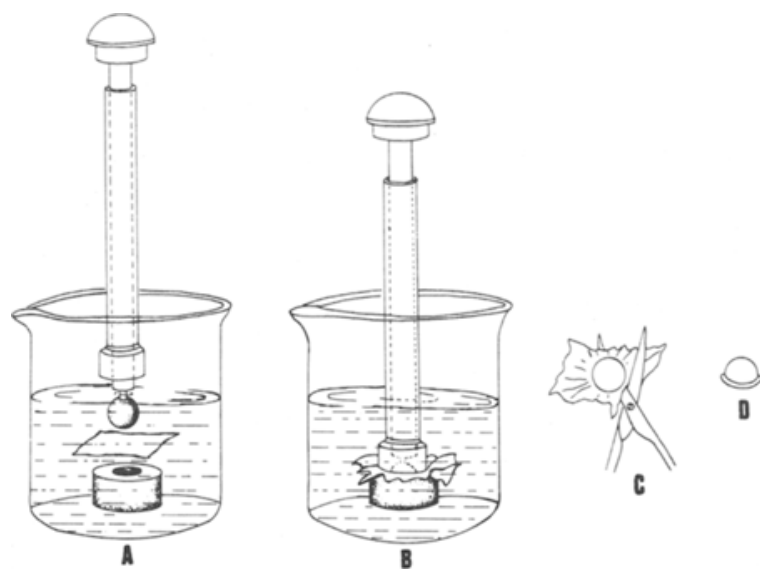

Figure 1. Illustration of method of stamping and trimming occluders. 


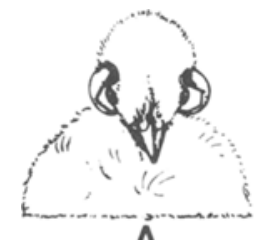

A

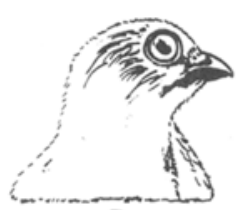

B

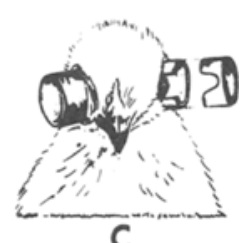

C

Figure 2. Birds wearing occluders: Plastic occluders can restrict bird to frontal vision (A) or prevent form vision in one eye (B). Two-piece metal occluder (C) may be used to completely prevent vision (on bird's right eye) or to restrict vision to one part of the visual field (on bird's left eye-exploded view). This occluder permits rapid interchange of visual fields.

with boiling water. A square of vinyl is held over the mold with a hemostat, and the ball end of the stamper is positioned to rest on the vinyl over the hole in the cylinder. The collar on the sliding sleeve is held down tightly to keep the rim from developing wrinkles or pleats, and the occluder is formed by gently pushing down on the rounded handle of the aluminum plunger. Once removed from the hot water, excess vinyl is trimmed to leave a narrow (1- to $2-\mathrm{mm}$ ) rim.

Domes of white plastic ranging in size from 10 to $15 \mathrm{~mm}$ in diameter can be produced in this manner, using stamping tools of different diameters. The finished product is glued over one eye to prevent any form vision in that eye (Figure $2 b$ ), without attenuating the light transmitted by more than .3 log units. To make an occluder that permits frontal but not lateral vision, a trapezoidal notch is cut in the side of the occluder (Figure 2a). To permit only lateral vision, a sector is sliced off the small end of the occluder. Notched occluders of more than $10-\mathrm{mm}$ diam should be made out of a material less flexible than .02 -in. vinyl; the material ping-pong balls are made of is satisfactory.

\section{METAL OCCLUDERS}

For experiments in which it is desirable to either alternate the eye being occluded or vary the available part of the visual field, we use a two-piece metal occluder. This consists of a notched ring fixed to the bird's head, permitting normal vision, and a cap that fits over this piece. A cap with a closed end may be placed over the notched ring to prevent any vision in that eye (Figure 2c, right eye). Once both eyes are outfitted with stationary inner rings, the cap may be switched from one eye to the other in a few seconds. Small modifications of the cap permit one to quickly change the part of the visual field the animal is using. A notch or hole of any desired size may be drilled in the side wall of the cap, and the bird can be given the desired amount of frontal vision by putting on the cap with the holes aligned (Figure 2c, left eye), or denied frontal vision by putting on the cap with holes not aligned. Exchanging this cap for one without an end cover

changes the animal's visual field from frontal to lateral. More precise control of the visual direction permitted can be obtained by making the hole in the cap much smaller than the other hole and rotating the cap appropriately. A series of dents around the cap permit seating at a variety of angles.

The pieces are made of telescoping $1 \mathrm{~cm}$ lengths of thin-walled brass tubing, with notches or holes drilled or ground in the wall. The end of the outer, removable length of tubing is covered with an epoxy-cemented piece of opaque vinyl. Small Parts, Inc., Miami, Florida, sells a line of thin-walled brass tubing with a wide range of diameters, each size telescoping in the next. To prevent the tube from collapsing during cutting, an abrasive cutting wheel, like those available for Dremel tools, is useful.

\section{DISCUSSION}

Plastic domes can be produced quickly, easily, and in sizes to suit large or small birds. We use $10-\mathrm{mm}$ diam occluders for newly hatched chicks and replace them with larger sizes as the birds grow. Collodion provides an easy means of repair should an occluder become loosened. The plastic occluders cannot be removed often, since some feathers are always removed with the occluder, leaving fewer feathers for the next attachment. With repair and replacement of these occluders, we have been able to sustain monocular visual deprivation in birds from the day of hatching to 6 weeks of age.

Compared to plastic occluders, brass occluders are more difficult to make and to use. Being heavier, they are not tolerated as well by the birds and are easier for the birds to remove. When using them with adult chickens, it was frequently necessary to hobble the chickens by tying a cord to each leg to make it difficult for them to scratch at the occluder. This was never necessary with the plastic occluders.

The occluders described here have a major advantage over contact lenses and hoods in that, since the occluder is glued to the skin around the eye, careful visual inspection of the boundary between the occluder and the skin permits one to determine whether any gaps exist through which the bird can see.

While this simple inspection is sufficient to insure that the birds cannot see around the edges of the occluders, we had some doubts as to whether the translucent plastic occluders actually prevented all form vision. A behavioral experiment was performed in which we compared the strength of the optokinetic nystagmus (OKN) with the occluder to that shown with a small hole in the occluder, and no occluder.

Eye movements were recorded by means of a fine coil of wire attached to the sclera of the eye, with the bird placed in a $25 \mathrm{kHz}$ alternating magnetic field. The signal from the coil was demodulated by a phase- 


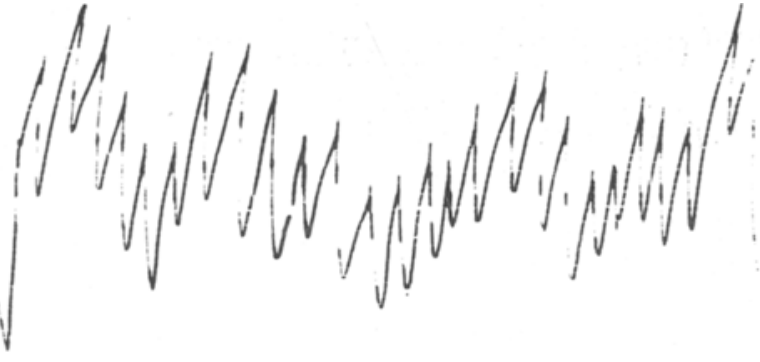

Eye Open

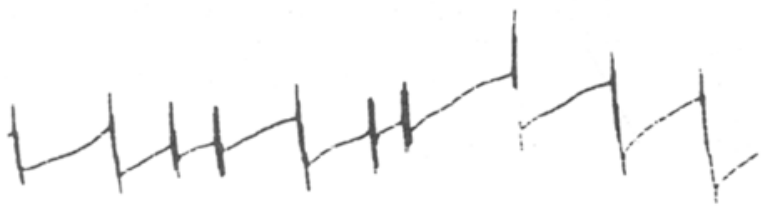

Occluder with $1.8 \mathrm{~mm}$ hole

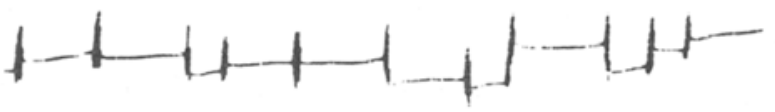

Occluder

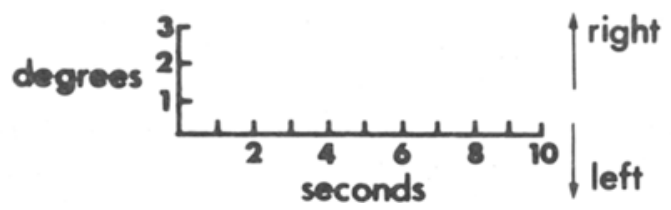

Figure 3. Effect of translucent occluder on optokinetic nystagmus. Note absence of nystagmus with transiucent occluder, whereas even a small hole substantially restores the $\mathrm{OKN}$. The cylinder rotated clockwise at $30 \mathrm{deg} / \mathrm{sec}$. This record is from the left eye. The slight rightward drift in the translucent occluder record is not OKN, but was also shown in the dark. sensitive detector and written out on a polygraph.

The lids of the recorded eye were held open with collodion; the other eye was covered. The bird was placed in the middle of a vertically striped drum in which each stripe pair subtended about $10 \mathrm{deg}$. The drum rotated clockwise at $30 \mathrm{deg} / \mathrm{sec}$.

The results (Figure 3 ) show that the strong OKN present without the occluder is abolished by the translucent occluder and largely restored by even a small hole in the occluder.

\section{REFERENCES}

Catania, A. C. Techniques for the control of monocular and binocular viewing in the pigeon. Journal for the Experimental Analysis of Behavior, 1963, 6, 627-629.

Cherkin, A. Eye to eye transfer of an early response modification in chicks. Nature, 1970, 227, 1153.

Chiu, P. S. L., Lauber, J. K., Kinnear, A. Dimensional and physiological lesions in the chick eye as influenced by the light environment. Proceedings of the Society for Experimental Biology and Medicine, 1975, 148, 1223-1228.

Hess, E. Space perception in the chick. Scientific American, 1956. 195, 71 .

Lauber, J. K., McGinnis, J., Boyd, J. Influence of miotics, diamox and vision occluders on light-induced buphthalmos in domestic fowl. Proceedings of the Society for Experimental Biology and Medicine, 1965, 120, 572-575.

Rossi, P. J. Adaptation and negative after-effect to lateral optical displacement in newly hatched chicks. Science, $1968,60,430-432$.

Siegel, A. I. Deprivation of visual form definition in the ring dove: II. Perceptual-motor transfer. Journal of Comparative and Physiological Psychology, 1953, 46, 249-252.

ZeIER, H. Lack of eye to eye transfer of an early response modification in birds. Nature, 1970, 225, 708-709.

(Received for publication November 10, 1977; revision accepted April 13, 1978.) 